\title{
DETERMINATION OF VAPOR PRESSURE FOR 4,4'-BIPYRIDINE BY GAS SATURATION AND GAS CHROMATOGRAPHIC METHODS
}

\author{
HSIEN-WEN KUO, JUNG-DER WANG* AND JIA-MING LIN
}

\author{
Center for the Researcher of Environmental and Occupational Diseases \\ National Taiwan University College of Medicine, Institute of Public Health, Taiwan, \\ Republic of China No.1, Section 1. Jen-Ai Road, Taipei, Taiwan, R.O.C.
}

\begin{abstract}
Vapor pressures of 4,4'-bipyridine were determined by gas saturation and gas chromatographic (GC) methods. The former agrees with the latter within 2 folds by a reference compound of pyridine. We recommend the GC method be performed with a column of similar polarity and a reference compound of similar structure and polarity.
\end{abstract}

\section{INTRODUCTION}

4,4'-bipyridine is a major intermediate product in paraquat manufacturing ${ }^{(1.2)}$. It was suspected of causing premalignant and malignant skin lesions on workers who have worked in the centrifugation and crystallization of bipyridine ${ }^{3}$. Also, 4,4'-bipyridine may be harmful to humans if inhaled, ingested or absorbed through the skin ${ }^{4}$. Kuo et al. ${ }^{5}$ recently developed and evaluated an air sampling method, which used an XAD-2 tube ${ }^{\circledR}($ SKC Inc., Eighty Four, P.A., U.S.A.) for the collection of 4,4'-bipyridine vapor.

4,4'-Bipyridine dihydrate exists as bitter needle form, with a melting point (mp) of $73{ }^{\circ} \mathrm{C}$, and a boiling point of $304.8^{\circ} \mathrm{C}$. It is freely soluble in alcohol, benzene, chloroform, diethyl ether; and slightly soluble in water ${ }^{6}$. However, we have searched several relevant data bases including Chemical Abstracts

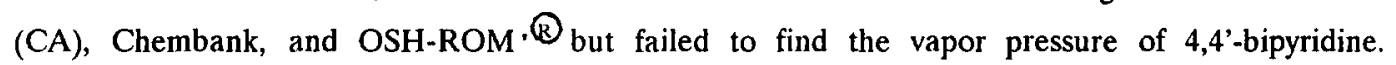
Unfortunately, paraquat is still used extensively as a herbicide in Taiwan because of its superior herbicidal effect ${ }^{7}$. A substantial atmospheric dissemination of bipyridyls can come from the direct 
handling of these intermediates during paraquat manufacturing or accidental spills. Distribution into the atmosphere is dependent on the vapor pressure and characteristics of its aerosol of bipyridyls and their affinities for solids or liquids. The objective of this study is to determine and verify the vapor pressure of 4,4'-bipyridine through GS and GC-calculated method. In addition, we also compare two different reference compounds in our GC-calculated methods.

\section{EXPERIMENTAL}

Materials: 4,4'-bipyridine (purity 98\%, Aldrich Chemical Co., Wisconsin, U.S.A.), and pyridine (Merck \& CO. Inc., Rahway, N.J., U.S.A.) were used without further purification. Methanol (MeOH, LC grade) and deionized water $\left(\mathrm{H}_{2} \mathrm{O}\right)$ were purified by Millpore filtration (Waters Associates Inc., Milford, M.A., U.S.A.). Diethyl ether (Merck) was the highest grade available commercially. XAD-2 tubes (mass of sorbent $50 / 100 \mathrm{mg}$, catalog No 22630-04, SKC, Inc.) were used as solid sorbent because of its high collection efficiency for the vapor of 4,4 '-bipyridine.

\section{Instrumentation Quantitative Measurements}

Gas saturation measurements were made by passing pure nitrogen gas at various flow rates through the gas-saturation apparatus (figure 1), maintained at $20,40,60$ and $80^{\circ} \mathrm{C}$. The evaporated gas was collected by XAD-2 tubes. The required amount of 4,4'-bipyridine was dissolved in $\mathrm{MeOH}$ and coated onto $1 \mathrm{~mm}$ diameter glass beads, and then packed into a clean U-shaped glass column (80 $\mathrm{cm}$ long * $0.6 \mathrm{~cm}$ i.d.). Initially, the dry cylinder air was slowly pumped through the column for 48 hours and gently agitated to evaporate any residual solvent before this experiment was performed. Carrier gas flow rates were regulated by a double needle gauge that was calibrated with a soap-bubble flowmeter and maintained at a constant flow rate of $4 \mathrm{ml} / \mathrm{min}$. The gas flow rate was monitored continuously with a flow meter (accuracy $0.5 \%$, range $1-200 \mathrm{ml} / \mathrm{min}$, Gilian Instrument. Corp., Wayne, N.J., U.S.A.). To determine if the condition of gas saturation was achieved, we plotted the vapor density of 4,4'-bipyridine as a function of residual time of carrier air in the U-shaped glass column ${ }^{8}$. Because of the low vapor pressure of $4,4^{\prime}$-bipyridine, it usually takes a long time to collect sufficient amounts for analysis. Figure 2 shows a typical plot of vapor density and the contact time, which indicates the condition of gas saturation at high temperature. For instance, a collection time of 56.7 hours at $20^{\circ} \mathrm{C}$ would be required to transpire 2 ug of 4,4'-bipyridine under our typical experiment conditions. Analyses were performed on a high performance liquid chromatograph (HPLC) (Model 441, Waters 


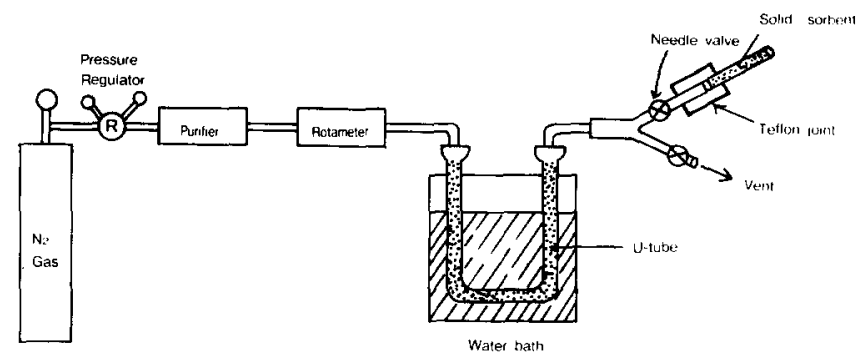

Figure 1. Gas Saturation apparatus

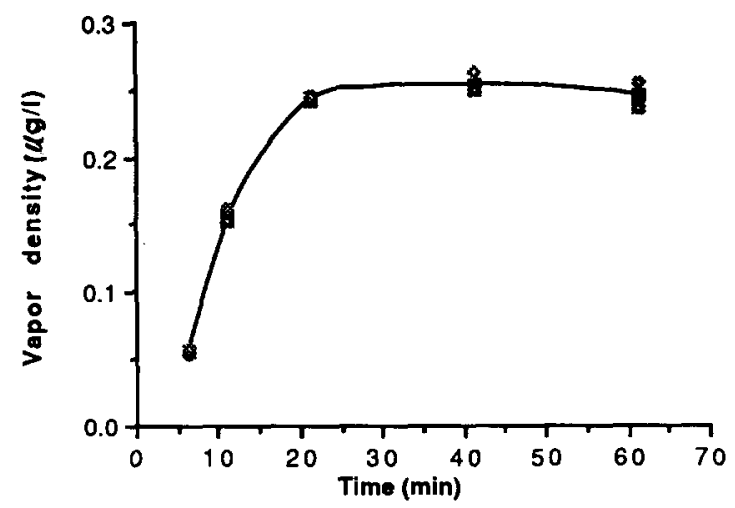

Figure 2. Vapor density of 4,4'-bipyridine as a function of contact time to air in $80^{\circ} \mathrm{C}$ temperature

Associates Inc.) equipped with UV detector at $254 \mathrm{~nm}$ wave length under the following conditions: mobile phase $50 / 50 \mathrm{MeOH} / \mathrm{H}_{2} \mathrm{O}$, flow $0.6 \mathrm{ml} / \mathrm{min}$, and chart speed $1 \mathrm{~cm} / \mathrm{min}$. The column was an Inertsi ${ }^{\mathrm{P}}$ ODS-2 (ID $4.6 * 150 \mathrm{~mm}$, Gasukuro Kagyo Inc., Tokyo, Japan). The absolute retention times for peak was $4.9 \mathrm{~min}$, and the instrument was tested for consistency by injecting ten pairs of high $(32.0$ $\mathrm{ug} / \mathrm{ml})$ and low $(1.0 \mathrm{ug} / \mathrm{ml})$ spiked concentrations of 4,4'-bipyridine, of which highly reproducible responses with coefficients of variation of $5.4 \%$ and $2.4 \%$ were obtained, respectively. Meanwhile, a suitable calibration standard was run for each injection concentration, and all calculations were 
performed with external standardization utilizing peak area response. A solid-phase vapor trap is usually not necessary to correct for any pressure difference between saturator and flow meter" The pressure drop across the XAD-2 tube was assumed to be negligible. The vapor pressure is calculated from the measured vapor density by using the ideal gas law ${ }^{(10.11)}$.

$$
P=d R T / M
$$

where $P$ is the vapor pressure, $d$ is the saturation vapor density, $M$ is the molecular weight, $T$ is absolute temperature and $\mathrm{R}$ is the gas constant.

\section{Gas chromatographic vapor pressure determination}

A gas chromatograph (HP model 5890) was used for measuring retention times of the 4,4'-bipyridine and reference compounds. The SE-30 packed column was operated under the following conditions; carrier gas $\mathrm{N}_{2}(40 \mathrm{ml} / \mathrm{min})$, injection temperature $260{ }^{\circ} \mathrm{C}$ and detection temperature $250^{\circ} \mathrm{C}$. Vapor pressures of 4,4'-bipyridine and reference compounds were calculated by Hamilton's method ${ }^{12}$ using two reference compounds (dibutyl phthalate and pyridine) at the same temperature. The vapor pressures of test $\left(P_{1}\right)$ and reference $\left(P_{2}\right)$ substances are related through the following equations:

$$
\begin{aligned}
& \ln T_{r} / T_{r_{2}}=\left(1-L_{1} / L_{2}\right) \ln P_{2} \cdot C \text { (I) } \\
& \ln P_{i}=\left(L_{1} / L_{2}\right) \ln P_{2}+C
\end{aligned}
$$

where $L$ is the latent heat of vaporization, $C$ is a constant, $T_{r 1}$ is the retention of GC for the test compound, and $T_{r 2}$ is the retention time of the reference compound. The relationship of $\ln P_{1}$ and $\ln$ $P_{2}$ at different temperatures was obtained from the slope $\left(1-L_{1} / L_{2}\right)$ and intercept $C$ by linear regression. Then, the vapor pressure $\left(P_{1}\right)$ of the test compound at $25^{\circ} \mathrm{C}$ was calculated by equation 2. When the compound with a melting point higher than the calculated temperature and lower than GC temperature was used, a melting point correction was applied using the equation $(3)^{(13.14)}$

$$
\ln P_{s} / P_{i}=6.79\left(l-T_{i i} / T\right)
$$

where $P_{s}$ and $P_{1}$ are vapor pressure for the solid and the liquid and $T_{n}$ and $T$ are the absolute melting point and the temperature at which the vapor pressure is calculated, respectively. The relationship between $\log \mathrm{P}$ and $1 / \mathrm{T}$ should be linear unless a change of state or a transition point occurs within the measured temperature range. The simplest, and certainly most successful, equation was developed by Antoine $\mathrm{e}^{(10.11)}$.

$$
\log P=A \cdot B / T
$$




\section{RESULTS AND DISCUSSION}

\section{Vapor pressure of the GS method}

The desorption efficiency of XAD-2 for 4,4'-bipyridine over the range of 10 to 222.4 micrograms was found $97.5 \%$, and the collection efficiency was $96.5 \%$ using a bubbler-U-tube sorbent train ${ }^{5}$. An analysis of the back section of each XAD-2 tube was performed to guarantee no breakthrough. Vapor pressures of 4,4'-bipyridine at different temperatures by gas saturation are summarized in Table $\mathbf{I}$. Each experiment was performed in triplicate with relatively small standard deviations. Because there has been no published data on vapor pressures of 4,4'-bipyridine, we used the Watson's approximation $^{15}$ to estimate the vapor pressure of $4,4^{\prime}$-bipyridine for comparison. Below the melting point $\left(73{ }^{\circ} \mathrm{C}\right)$, the two values were in relatively good agreement.

Table 1. Comparison of 4,4'-bipyridine vapor pressure vapor pressure by GS (gas saturation) method with Watson's approximation at various temperature

\begin{tabular}{cccc}
\hline Temperature $\left({ }^{\circ} \mathrm{C}\right)$ & GS method & $(95 \%$ C.I. $)$ & Watson's approximation \\
\hline 20 & $5.93 * 10^{-5}$ & $(5.81-6.05)$ & $6.40^{*} 10^{-5}$ \\
40 & $5.82 * 10^{-4}$ & $(5.76-5.88)$ & $6.81 * 10^{-4}$ \\
60 & $6.03 * 10^{-3}$ & $(5.98-6.09)$ & $6.13^{*} 10^{-3}$ \\
80 & $3.49 * 10^{-2}$ & $(3.39-3.59)$ & $1.49 * 10^{-1}$ \\
\hline
\end{tabular}

* $95 \%$ confidence limit calculated with the equation $2 \mathrm{Sm}=2 \mathrm{SD} / \sqrt{\mathrm{n}}$, where $\mathrm{S}$ standard deviation and $\mathrm{n}$ : number of determination

\section{Vapor pressure of the GC method}

Several operational parameters of the gas chromatograph might influence retention data, and thus, vapor pressures calculated from the retention data. Table 2 showed different calculated vapor of 4,4'-bipyridine by GC retention data using DBP and pyridine as the reference compounds. If data obtained by the GS method was ideal, we found that vapor pressures estimated by the GC-calculated method were influenced by the mixture of reference compounds and melting point correction. In fact, 4,4'-bipyridine vapor pressures estimated from pyridine as the reference compound with a mp correction seemed so accurate that they were usually less than 2 folds of difference compared to the 
GS method (Table 2). Although DBP has been used as a general reference compound for the determination of vapor pressure in previous experiments ${ }^{(12.13)}$, our results showed that the slope of $\log$ $P_{G C}$ (vapor pressure estimated from gas chromatographic method) vs $1 / T$ was more negative and all points fell below the GS method except at the high temperature in figure 3 . Alternatively, $P_{G C}$ with pyridine as a reference compound agreed better with the GS method.

Table 2. Comparison of GC-calculated vapor pressures (torr) with experimental values obtained from the gas saturation.

The GC data were stratified by different reference compounds and with or without melting point correction.

\begin{tabular}{|c|c|c|c|c|c|}
\hline \multirow[t]{2}{*}{ Temp $\cdot\left({ }^{\circ} \mathrm{C}\right)$} & \multirow[t]{2}{*}{$\begin{array}{l}\text { Experimental } \\
\text { valus (GS) }\end{array}$} & \multicolumn{2}{|c|}{$\begin{array}{c}\text { GC-calculated values } \\
\text { Dibutyl Phthalate }\end{array}$} & \multicolumn{2}{|c|}{$\begin{array}{l}\text { GC-calculated values } \\
\text { Pyridine } \\
\end{array}$} \\
\hline & & No mp corr. & With mp con. & No mp corr. & With mp corr. \\
\hline 20 & $5.56^{*} 10^{-5}$ & $3.54^{*} 10^{-5}$ & $4.00 * 10^{-6}$ & $2.63 * 10^{-4}$ & $2.97^{*} 10^{-5}$ \\
\hline 40 & $6.29 * 10^{-4}$ & $3.03^{*} 10^{-4}$ & $6.06 * 10^{-5}$ & $2.49^{*} 10^{3}$ & $4.70^{*} 10^{-4}$ \\
\hline 60 & $5.31 * 10-3$ & $2.01 * 10^{3}$ & $6.66^{*} 10^{-4}$ & $1.70^{*} 10^{2}$ & $5.37^{*} 10^{-3}$ \\
\hline 80 & $3.52 * 10^{-2}$ & $9.01^{*} 10^{2}$ & $4.67^{*} 10^{?}$ & $8.96^{*} 10^{-2}$ & $4.65^{*} 10^{2}$ \\
\hline
\end{tabular}

GS: Gas saturation method

The calculation of GC method is based on the assumption that the vapor pressure is inversely proportional to the relative retention times of the chemical ${ }^{(12.16)}$. However, the retention time of a compound usually depends on other factors, such as the relative polarity of the test compound and the packed material in the GC column. Since the column (silicone SE-30) we used was relatively nonpolar, DBP as a relatively polar compound (dipole moment 2.4), was eluted quickly from the column compared to 4,4'-bipyridine (dipole moment 0.6 ). The relative retention time of DBP to 4,4'-bipyridine would generally be biased toward 0 , which leads to underestimation of the vapor pressure of $4,4^{\prime}$-bipyridine as we found in Table 2 . While pyridine is not only more nonpolar in nature (dipole moment 2.2) but also very similar to 4,4'-bipyridine in structure, it generally fulfills the original assumption of the GC calculation method that other determinants of the retention time should be held relatively constant, and it produced a very close approximation to the GS method. We thus conclude that a column with similar polarity and a reference compound of similar structure \& polarity would 
contribute to the accuracy of the GC calculation method.

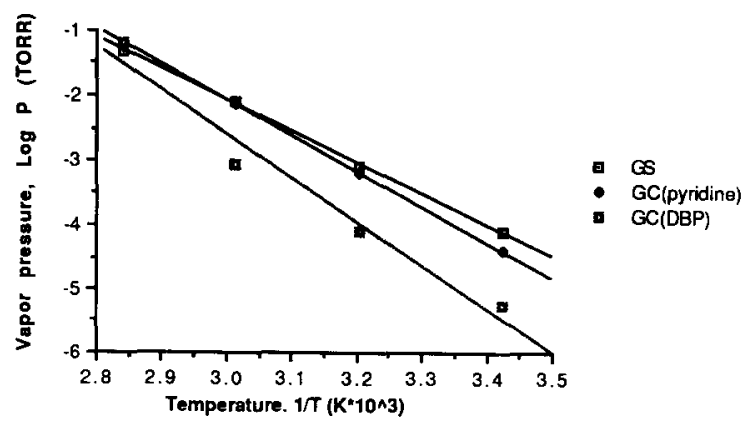

Figure 3. Logarithms of vapor pressure and 1/Temperature (1/T) plotted according to the Antoine equation for 4,1-bipyrdine obtained by different methods: GS (gas saturation) GC (gas chromatographic method) DBP (Dibutyl phthalate)

We also evaluated the influence of carrier gas flow in GC-calculated method with three different speeds of flow rate. Theoretically, there should be no difference in the calculated vapor pressures. However, Table 3 shows that a lower flow rate consistently leads toward a slightly higher vapor pressure, which is close to the GS method. We attribute this effect to the relatively low vapor pressure of 4,4'-bipyridine.

Table 3. Comparison of GC-calculated vapor pressure (torr) different carrier gas flow in 4,4'-bipyridine

\begin{tabular}{cll}
\hline Temperature $\left({ }^{\circ} \mathrm{C}\right)$ & low $(14.2 \mathrm{cc} / \mathrm{ml})$ & high $(43.8 \mathrm{cc} / \mathrm{ml})$ \\
\hline 20 & $2.97^{*} 10^{-5}$ & $2.68 * 10^{-5}$ \\
40 & $2.68^{*} 10^{-4}$ & $4.26^{*} 10^{-4}$ \\
60 & $5.37^{*} 10^{-3}$ & $4.87 * 10^{-3}$ \\
80 & $4.65^{*} 10^{-2}$ & $4.22^{*} 10^{-2}$ \\
\hline
\end{tabular}

* Pyridine was reference compound

Antoine plots of $\log \mathrm{P}$ vs $1 / \mathrm{T}$ were obtained for $4,4^{\prime}$-bipyridine by GS and GC methods with pyridine and DBP as two independent reference compounds (Figure 3 ). The plot from the GS method agreed very closely with that calculated by the GC method using pyridine as a reference compound. Slopes and intercepts of these other two plots were -4.83 and $-5.52,12.2$ and 14.3, respectively, and correlation coefficients both were 0.999 . Therefore, the heat of vaporization $(\Delta \mathrm{Hv})$ of 4,4 -bipyridine can be calculated by the following equation ${ }^{17}$

$$
\Delta H v=-(2.303) R M
$$


where $R$ is the gas constant and $M$ is the slope of the line. The $\Delta H$ v's $^{\prime}$ of $4,4^{\prime}$-bipyridine were 22.1 and $25.3 \mathrm{Kcal} / \mathrm{mol}$, respectively.

\section{Conclusion}

Vapor pressures of 4,4'-bipyridine determined by gas saturation method agree within 2 folds with that calculated by the gas chromatographic method using pyridine as a reference compound. We conclude that to improve the accuracy of the GC calculation method for estimation of vapor pressure it should be performed with a column of similar polarity and a reference compound of similar structure \& polarity.

\section{REFERENCE}

(1). Bowra,G.T., Duffield,D.P., Osborn,A.J. Br. J. Ind. Med. 39,76-81 (1982).

(2). Li,W.M. Dermatological Sinica 2,1-8 (1984).

(3). Wang,J.D., Li,W.M., Hu,F.C., Hu,K.H. Br. J. Ind. Med. 44,196-200 (1987)

(4). Hayes, W.J. " Pesticides Studies in Man", Williams \& Wilkns (1982).

(5). Kuo,H.W., Wang,J.D., Lin,J.M. Am. Ind. Hyg. Assoc. J. (1991) (revised).

(6). The Merck Index. Merck \& CO., Inc. 14 edition (1983).

(7). Council of Agricuture. Introduction to Pesticides in Taiwan. Taipei, Taiwan: Executive Yuan, R.O.C., (1989),1-17.

(8). Westcott,J.W., Simon,C.G. and Bidleman,T.F. Enviro. Sci.\& Tech. 15,1375-1378 (1981).

(9). Spencer,W.F., Cliath,M.M. Residue Review. 85,58-71 (1983).

(10). Thomson,G.W. "Techniques of Organic Chemistry", in Weissberger and Roissiter Vol I, Part I Wiley-Interscience (1971).

(11). Nesmeyanov,A.N. "Vapor Pressure of the Chemical Elements", Eleevier Publishing Company (1959).

(12). Hamilton,D.J. J. of Chromatogr. 195, 75-83 (1980).

(13). Bidleman, T.F. Anal. Chem. $\underline{56}, 2490-2496$ (1984).

(14). Eitzer,B.D. and Hites,R.A. Enviro. Sci. \& Tech. 22,1362-1364 (1988).

(15). Grain,C.F. "computer aided chemical thermodynamic gas and liquid. theory, models and program", in Paul, B and Francis O, John Wilk \& Sons (1985).

(16). Westcott,J.W. and Bidleman,T.F. J of Chromatogr. 314,37-53 (1984).

(17). Spencer,W.F., Cliath,M.M. Enviro. Sci. \& Tech. 3, 670-4 (1969).

(Received in Germany 24 March 1992; accepted 14 April 1992) 